\title{
Structure-Guided D0T1L Probe Optimization by Label-Free Ligand Displacement
}

\section{Citation}

Yi, J., A. J. Federation, J. Qi, S. Dhe-Paganon, M. Hadler, X. Xu, R. St. Pierre, et al. 2014. "Structure-Guided DOT1L Probe Optimization by Label-Free Ligand Displacement." ACS Chemical Biology 10 (3): 667-674. doi:10.1021/cb500796d. http://dx.doi.org/10.1021/cb500796d.

\section{Published Version}

doi:10.1021/cb500796d

\section{Permanent link}

http://nrs.harvard.edu/urn-3:HUL.InstRepos:23845221

\section{Terms of Use}

This article was downloaded from Harvard University's DASH repository, and is made available under the terms and conditions applicable to Other Posted Material, as set forth at http:// nrs.harvard.edu/urn-3:HUL.InstRepos:dash.current.terms-of-use\#LAA

\section{Share Your Story}

The Harvard community has made this article openly available.

Please share how this access benefits you. Submit a story.

\section{Accessibility}




\title{
Structure-Guided DOT1L Probe Optimization by Label-Free Ligand Displacement
}

\author{
Joanna S. Yi, ${ }^{\dagger, \ddagger, \S}$ Alexander J. Federation, ${ }^{\dagger}$ Jun Qi, $^{\dagger}{ }^{\downarrow}$ Sirano Dhe-Paganon, ${ }^{\|}$Michael Hadler, ${ }^{\perp}$
} Xiang Xu, ${ }^{\#}$ Roodolph St. Pierre, ${ }^{\dagger}$ Anthony C. Varca, ${ }^{\dagger}$ Lei Wu, ${ }^{\bigcirc}$ Jason J. Marineau, ${ }^{\dagger}$ William B. Smith, ${ }^{\dagger}$ Amanda Souza, ${ }^{\dagger}$ Emma J. Chory, ${ }^{\dagger}$ Scott A. Armstrong, ${ }^{\perp}$ and James E. Bradner* ${ }^{\dagger}, \nabla$

${ }^{\dagger}$ Department of Medical Oncology, Dana-Farber Cancer Institute, Boston, Massachusetts, United States

${ }^{\ddagger}$ Department of Pediatric Oncology, Dana-Farber Cancer Institute, Boston, Massachusetts, United States

${ }^{\S}$ Department of Pediatrics, Harvard Medical School, Boston, Massachusetts, United States

"Department of Cancer Biology, Dana-Farber Cancer Institute, Boston, Massachusetts, United States

${ }^{\perp}$ Human Oncology and Pathogenesis Program, Department of Pediatrics, Memorial Sloan Kettering Cancer Center, New York, New York, United States

\#Department of Biological Chemistry and Molecular Pharmacology, Harvard Medical School, Boston, Massachusetts, United States

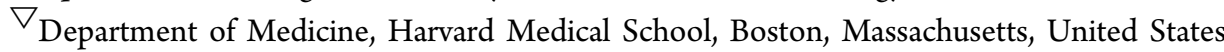

OShanghai ChemPartner Co. Ltd., 998 Hailei Road, Zhangjiang Hi-Tech Park, Pudong New Area, Shanghai, 201203, China

\section{Supporting Information}

ABSTRACT: The DOT1L lysine methyltransferase has emerged as a validated therapeutic target in $M L L$-rearranged (MLLr) acute leukemias. Although S-adenosylmethionine competitive inhibitors have demonstrated pharmacological proof-of-principle in $M L L$ r-leukemia, these compounds require further optimization to improve cellular potency and pharmacokinetic stability. Limiting DOT1L inhibitor discovery and ligand optimization have been complex biochemical methods often using radionucleotides and cellular methods requiring prolonged culture. We therefore developed a new suite of assay technologies that allows comparative assessment of chemical tools for DOT1L in a miniaturized format. Coupling these assays with structural information, we developed new insights into DOT1L ligand binding and identified several functionalized probes with increased cellular potency $\left(\mathrm{IC}_{50}\right.$ values $\sim 10$ $\mathrm{nM}$ ) and excellent selectivity for DOT1L. Together these assay technologies define a platform capability for discovery and optimization of small-molecule DOT1L inhibitors.
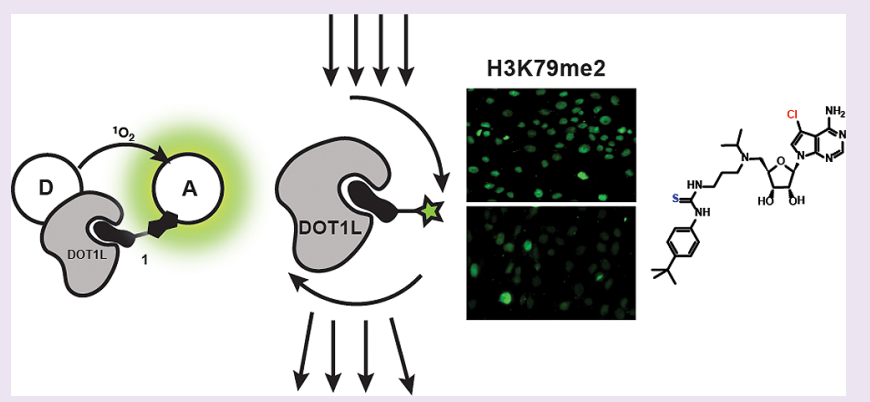

Mixed-Lineage Leukemia ( $M L L)$ gene rearrangements occur in $5-10 \%$ of all acute leukemia patients and in greater than $70 \%$ of infants with acute lymphoblastic leukemia (ALL). The presence of the rearrangement portends a poor prognosis, despite aggressive therapy with significant associated morbidity. ${ }^{1}$ Cooperation between specific chromatin-modifying complexes and $M L L$-rearranged gene products defines disease pathogenesis and has prompted efforts to target modulators of chromatin structure and function in this cancer. ${ }^{2,3}$

MLL is a member of the Trithorax family of proteins and functions as a histone lysine methyltransferase (KMTase). ${ }^{1}$ During development, MLL catalyzes trimethylation of lysine 4 on histone 3 from the methyl donor S-adenosylmethionine (SAM) at homeobox genes, promoting their expression. In MLL-rearranged leukemia, however, the SET domain responsible for KMTase activity is uniformly lost with translocation and replaced by one of more 70 known fusion partners. ${ }^{3}$ Many of these fusion partners recruit DOT1L, which is the only known methyltransferase responsible for the mono-, di-, and trimethylation of lysine 79 of histone 3 (H3K79). H3K79 methylation is associated with most actively transcribed genes and marks regions of elongating RNA Pol II typically within the first intron of gene bodies. ${ }^{4}$ The recruitment of DOT1L by MLL fusion partners to developmental MLL-target genes results in aberrant hypermethylation of $\mathrm{H} 3 \mathrm{~K} 79$ at these loci, contributing to leukemogenesis by inappropriately sustained gene expression, namely at the HOXA locus. ${ }^{5-8}$

The therapeutic significance of DOT1L in established MLLrearranged leukemia has been validated by genetic and chemical genetic approaches. Conditional inactivation of Dot1L in MLLAF9 (and AF6 and AF10) leukemia models results in diminished H3K79 methylation and prolonged survival. ${ }^{6,9-12}$

Received: October 3, 2014

Accepted: November 14, 2014

Published: November 14, 2014 
A

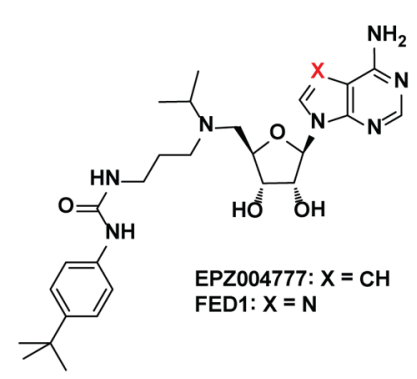

B

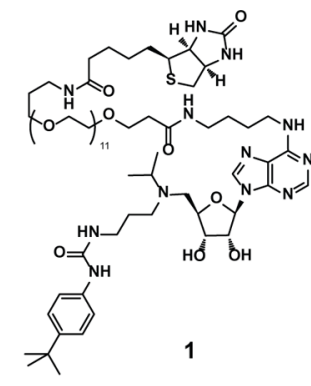

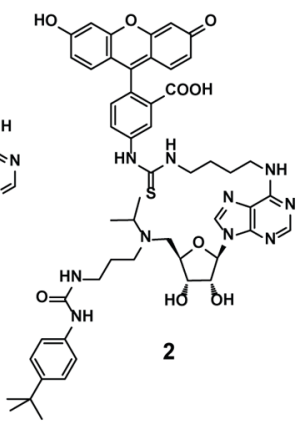

E

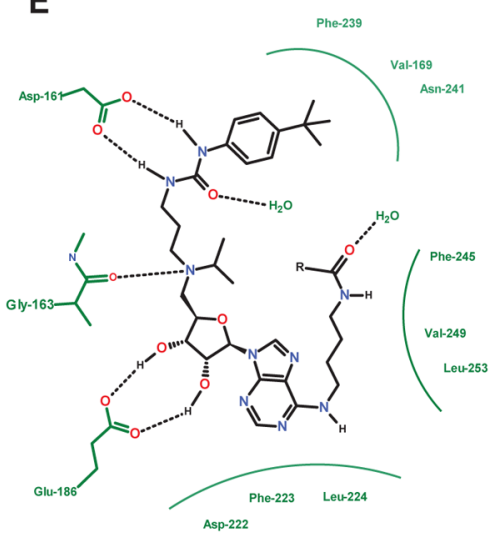

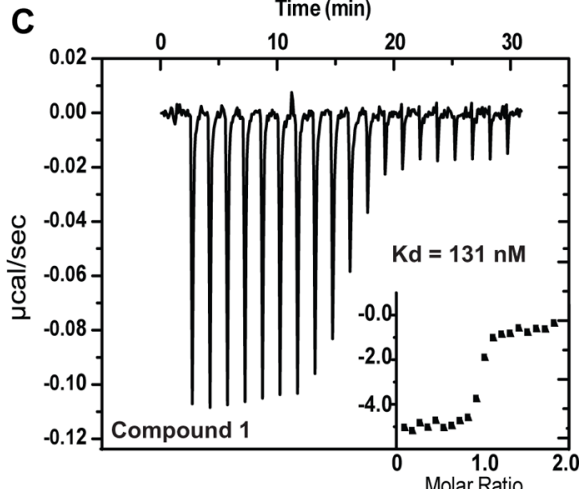

$\mathbf{F}$

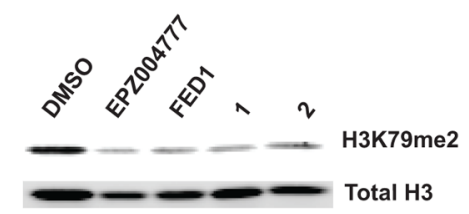

G

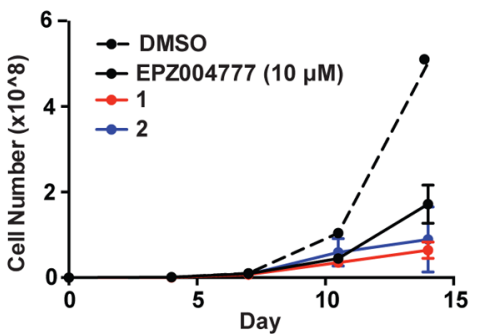

Figure 1. Design and characterization of chemical probes of DOT1L. (a) Structures of EPZ004777 ${ }^{13}$ and FED1. ${ }^{14}$ (b) Structures of biotinylated (1) and FITC-labeled (2) FED1 attached via a linker to the N6 position of the base. (c) Isothermal calorimetry analysis of 1 demonstrating strong 1:1 binding with DOT1L. (d) Binding of 1 to DOT1L demonstrates linker exposure and a similar binding mode to that of FED1. (e) Detailed ligandinteraction diagram of 1 demonstrating new hydrogen bond formation. (f) Inhibition of H3K79me2 by indicated DOT1L inhibitors (10 uM) in MV4;11 cells treated for 4 days. (g) DOT1L probe treatment results in inhibition of MV4;11 cell growth over time.

Recently, SAM-competitive small-molecule inhibitors of DOT1L have been developed, first reported in $2011,{ }^{13}$ and further characterized biochemically and structurally by our group in collaboration with Professor Cheryl Arrowsmith. ${ }^{14}$ Structurally, these SAM mimetics featured high potency and selectivity for DOT1L. However, the cellular activity of these compounds is rather low in potency relative to the extraordinary subnanomolar binding potency in homogeneous assays in vitro. Notably, the antileukemic effect of DOT1L inhibition requires 10-14 days of continuous dosing at high $(1-3 \mu \mathrm{M})$ concentrations in cell culture models using current inhibitors. ${ }^{14-19}$ In animal studies, this translates to a modest benefit in survival while requiring high doses through continuous osmotic subcutaneous infusion. ${ }^{13,15}$ Further optimization of DOT1L inhibitors is therefore needed.

To date, development of structurally divergent DOT1L inhibitors has been slow in the broader epigenetics community, perhaps relating to the challenges in biochemistry and cell biology platforms which underlie ligand discovery and optimization. Thus far, biochemical assays of DOT1L use radioligands and often require specialized synthetic or highly purified histone particles as substrates. Additionally, the ubiquitinylation of nucleosomes strongly influences DOT1L activity and poses difficulties to ligand discovery. ${ }^{20}$ The delayed cellular effects of DOT1L inhibition challenge the miniaturization of cell-based measures of compound potency. Simple dose-ranging comparisons have proven time-consuming and low-throughput. We therefore identified an opportunity to create a facile discovery platform enabling the characterization of existing DOT1L inhibitors, and the preparation of new compounds with improved properties. Herein, we report the development of tagged DOT1L ligands used in robust and miniaturized biochemical assays, as well as a high-throughput, high-content assay system that reports on pharmacodynamic H3K79 methylation abundance in short incubation windows. Together, these three orthogonal assays have defined a platform capable of discovering and optimizing novel DOT1L inhibitors.

\section{RESULTS AND DISCUSSION}

Toward the development of DOT1L chemical probes, we chose a SAM-competitive inhibitor from our laboratory (FED1) as a suitable starting point to develop assay ligands for DOT1L (Figure 1a). FED1 is a near chemical derivative of EPZ004777 that features a more efficient and high-yielding synthesis. ${ }^{14}$ Additionally, FED1 has a modestly reduced binding potency for DOT1L that was postulated to improve utility in competition binding assay development across a broad range of inhibitors. Given the extended residence times of DOT1L inhibitors (EPZ004777 $K_{\text {off }}=9.29 \times 10^{-4} \mathrm{~s}^{-1}$ and FED1 $K_{\text {off }}=$ $\left.2.20 \times 10^{-3} \mathrm{~s}^{-1}\right),{ }^{14}$ a less potent inhibitor such as FED1 may provide the opportunity to discover weaker initial assay positives in high-throughput screening. The crystal structure of FED1 shows a binding mode similar to EPZ004777, with the tert-butyl phenyl urea motif further extending the binding 
A

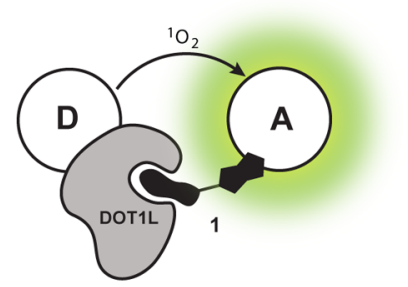

D

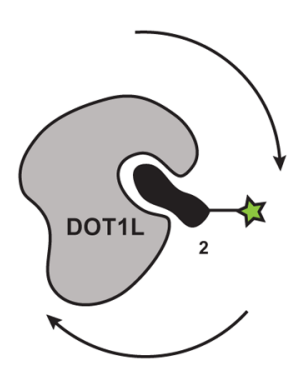

G

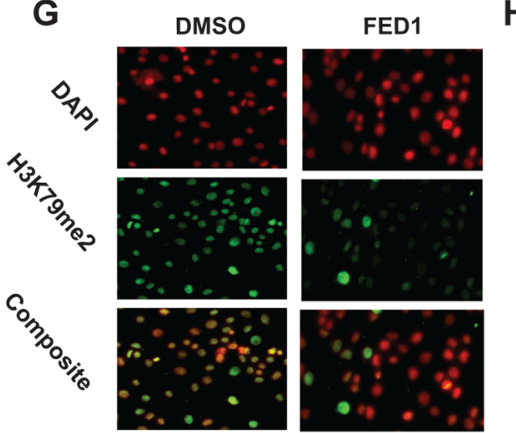

B

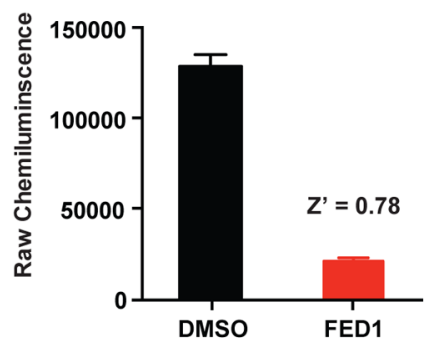

E

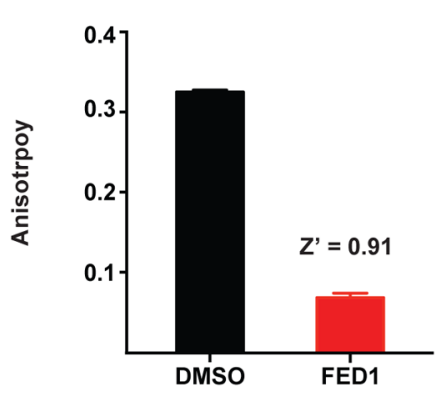

H

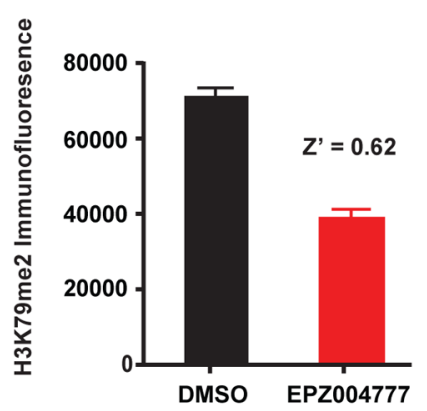

C

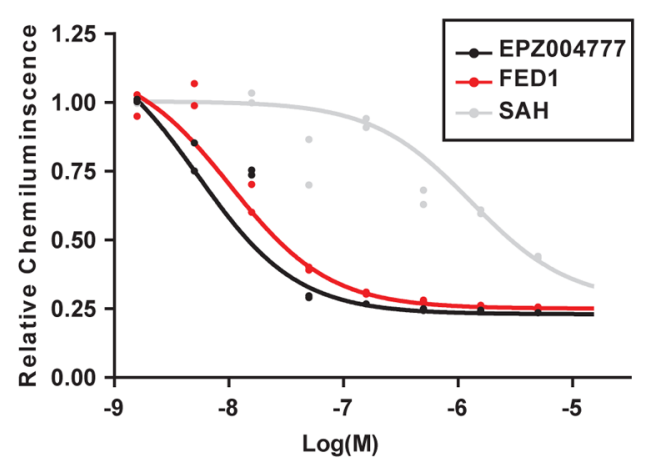

F
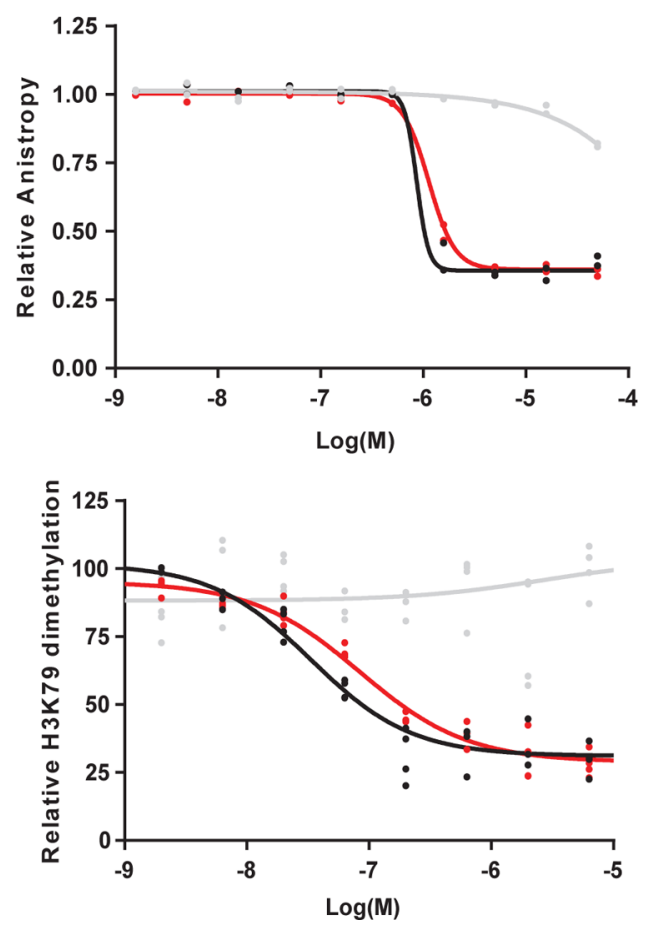

Figure 2. Development of nonradiometric biochemical and cellular assays for DOT1L. (a-c) AlphaScreen proximity bead-based assay demonstrating adaptability to high-throughput screening $\left(Z^{\prime}\right.$ calculated by $1-\left(\left(3 \sigma_{\mathrm{FED} 1}+\sigma_{\mathrm{DMSO}}\right) /\right.$ absolute value $\left.\left(\mu_{\mathrm{FED} 1}-\mu_{\mathrm{DMSO}}\right)\right)$, and expected comparable potency differentiation of known inhibitors. $(\mathrm{d}-\mathrm{f})$ Fluorescence polarization assay demonstrating significant assay robustness $\left(\mathrm{Z}^{\prime}\right.$ calculated with above formula) and separation of weak DOT1L inhibitors (SAH) from more potent compounds (FED1 and EPZ004777). (g-i) High-content imaging assay evaluating H3K79me2 abundance by immunofluorescence in A431 cells after 4 days of indicated DOT1L inhibitors, with diminished $\mathrm{H} 3 \mathrm{~K} 79 \mathrm{me} 2$ compared to DMSO. Assay is robust $\left(\mathrm{Z}^{\prime}\right.$ calculated as above) and reports cellular $\mathrm{EC}_{50}$. Biochemical assays were performed in duplicate, and high-content assays were performed as four replicates.

pocket compared to SAM (Figure S1a, PDB: 4ER0). ${ }^{14}$ While most of the molecule is deeply obscured in the binding pocket and inaccessible to solvent, the more open position of the nucleotide base suggested a tolerance for further chemical substitution. We postulated that modification on the N6 position of the FED1 adenine would not interfere with the activity of the molecule, allowing the installation of features with functional utility (e.g., retrievable chemical linkers and fluorophores).

We synthesized two probes (Supporting Information Schemes 1 and 2), 1 with polyethylene glycol (PEG) linked biotin and 2 with a thiourea-coupled fluorescein (FITC) (Figure 1b). The binding affinities of these two modified inhibitors were confirmed by isothermal titration calorimetry and are comparable to the parent compound (Figure 1c and Figure S1b). The apparent potency of $\mathbf{1}$ was also similar to the parent compound by differential scanning fluorimetry (Figure
S1d). The crystal structure of 1 with DOT1L was then obtained. Ligand $\mathbf{1}$ bound to the SAM pocket as expected, with the structured features of the linker protruding out toward the solvent. The lack of atomic density for the remaining PEG and biotin features likely reflects unrestricted mobility in solvent (Figure 1d). The ligand-interaction diagram further confirmed that DOT1L binds to 1 and FED1 in a manner dictated by common determinants of molecular recognition (Figure 1e, Figure S1c). The amide bond of the linker unit also formed a hydrogen bond with a structured water molecule, which may contribute to preservation of the ligand binding activity. Cell permeability was confirmed by immunoblot for the H3K79 dimethyl (H3K79me2) histone mark, which was efficiently depleted by both ligands (Figure 1f). As then expected, both compounds inhibited cell proliferation comparably to EPZ004777 after 10-14 days (Figure 1g). 
A
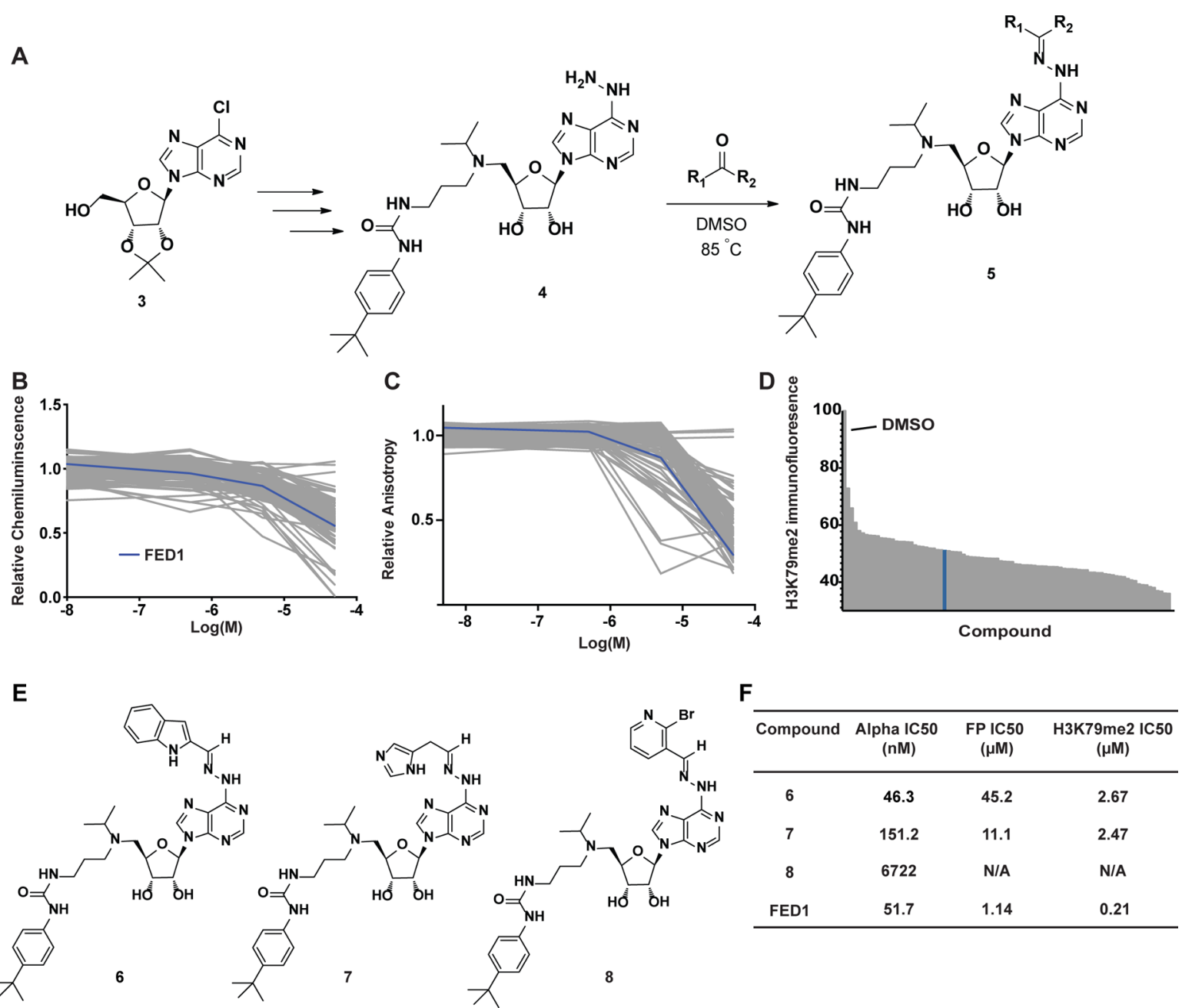

$\mathbf{F}$

\begin{tabular}{cccc}
\hline Compound & $\begin{array}{c}\text { Alpha IC50 } \\
(\mathrm{nM})\end{array}$ & $\begin{array}{c}\text { FP IC50 } \\
(\mu \mathrm{M})\end{array}$ & $\begin{array}{c}\text { H3K79me2 IC50 } \\
(\mu \mathrm{M})\end{array}$ \\
\hline 6 & 46.3 & 45.2 & 2.67 \\
7 & 151.2 & 11.1 & 2.47 \\
8 & 6722 & N/A & N/A \\
FED1 & 51.7 & 1.14 & 0.21 \\
\hline
\end{tabular}

Figure 3. Purine substitutions of adenosyl DOT1L inhibitors. (a) Synthetic scheme illustrating generation of focused library of hydrazine inhibitors, modified off the N6 position. (b, c) Biochemical screening results of the library at four doses, with (d) cellular H3K79me2 screen at 20 uM compared to FED1 (indicated in blue). (e) Structures of resynthesized assay positives. (f) Profiling table of validated assay positives compared to FED1.

With these validated ligands in hand, we next developed orthogonal biochemical assays capable of detecting small molecule binding to purified, recombinant human DOT1L protein. The first assay utilizes 1 and employs a nanomaterial proximity assay (AlphaScreen, PerkinElmer; Figure $2 a-c$ ). The biotin on 1 recruits a streptavidin-coated donor bead, while the FED1 portion of the molecule recruits a nickel-coated receptor bead via binding to the recombinant $\mathrm{HIS}_{6}$-DOT1L methyltransferase domain. Illumination of the donor bead releases singlet oxygen, which diffuses to activate in situ synthesis of a chemiluminescent lanthanide within the acceptor bead only when the two are in close proximity, here dependent on the DOT1L-ligand interaction. Displacement of 1 from DOT1L disrupts the proximity of the two beads and diminishes chemiluminescence. Finally, we have miniaturized the assay to microtiter plate format (384-well) and improved robustness compatible with high-throughput screening $\left(Z^{\prime}=0.78\right)$. Using a set of resynthesized DOT1L chemical tools, we confirmed faithful utility in comparative ligand potency determination ( $\mathrm{IC}_{50}$ for EPZ004777 $5.3 \mathrm{nM}$, FED1 22.4 nM, SAH $1299 \mathrm{nM}$ ).

Next, a fluorescence polarization (FP) assay was developed to monitor binding of inhibitors to DOT1L using the fluorescent probe 2 (Figure $2 \mathrm{~d}-\mathrm{f}$ ). After excitation with plane-polarized light, binding of 2 to DOT1L increases anisotropy of the bound state relative to free 2. Therefore, displacement of noncovalently bound 2 from DOT1L by a competitive ligand leads to a decrease in detectable signal. This FP assay has proven amenable to high-throughput screening $\left(Z^{\prime}=0.91\right)$ and accurately discriminates compounds in our reference set by relative potencies. In our experience to date, the FP assay is more suitable to HTS than the bead-based proximity assay owing to false-positives that are assay specific (nickel chelation, biotin mimetics). Together, these two assays are highly complementary in screening and lead optimization.

To support iterative development of DOT1L inhibitors using a cellular assay capable of reporting on methyltransferase function, we developed a high-content imaging assay to measure global nuclear H3K79me2 with short-term compound incubation (3-4 days; Figure 2g-i). The A431 epidermoid carcinoma cell line was selected based on high basal H3K79me2 and strong adherence, which tolerated the harsh immunofixation conditions required to detect staining of this mark on the histone body. Cells were treated for 4 days with the compound in 384-well plates and then assessed for H3K79me2 using primary and secondary (fluorescent-conjugated) antibodies. Using our reference compound set, the 


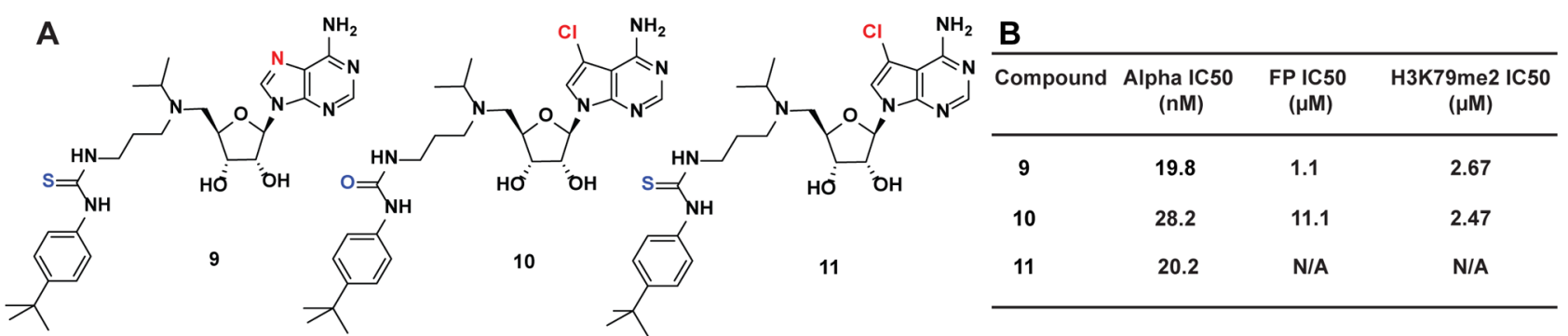

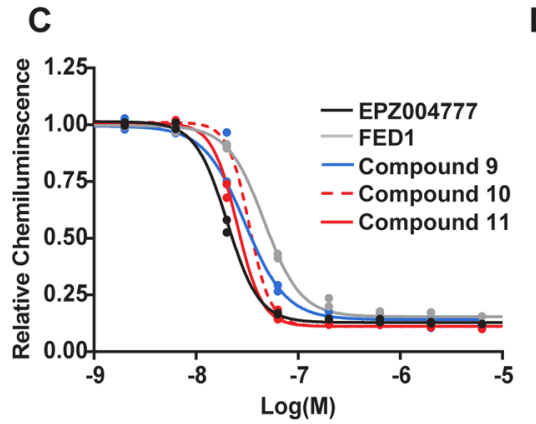

$\mathbf{F}$

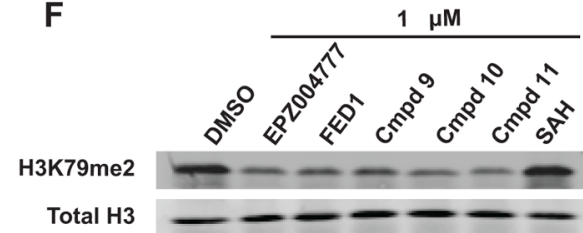

H

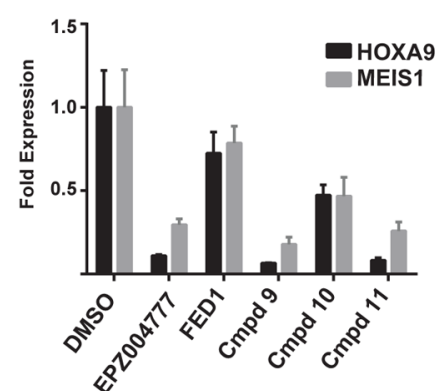

D

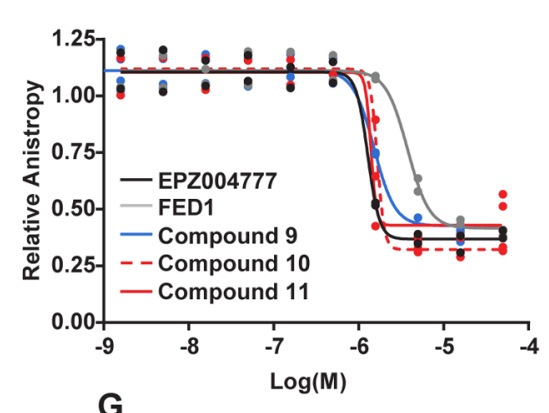

G

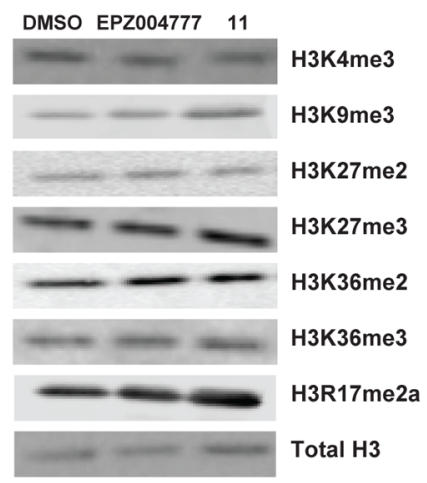

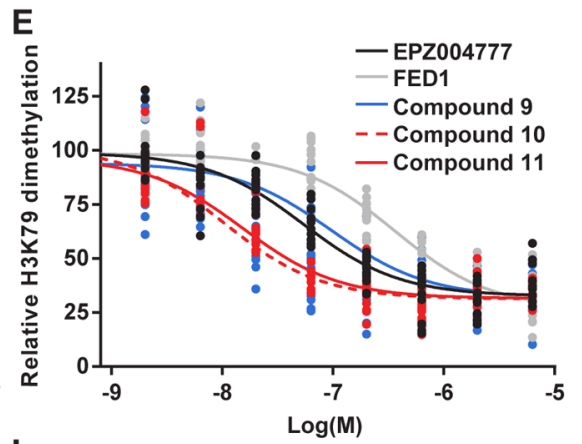
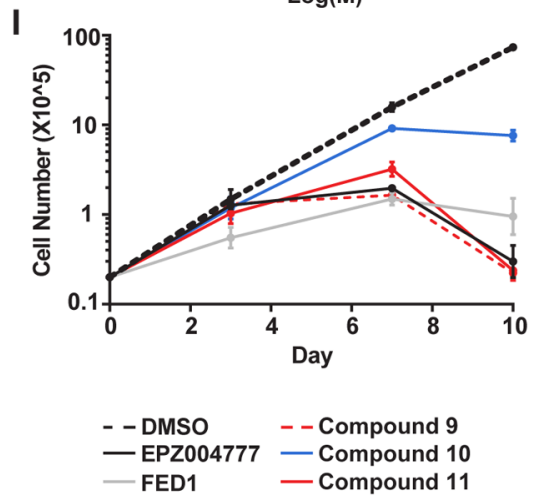

Figure 4. Development of potent chlorinated DOT1L inhibitors. (a) Structures of 9, 10, and 11. (b-e) Evaluation of SAM-like derivatives by biochemical assays demonstrating similar potency, and high content assay demonstrating increased potency of chlorinated inhibitors (10, 11) and improvement of FED1 potency with substitution of a thiourea tail. Biochemical assays were run in duplicate and high-content assays in quadruplicate. (9). (f) Immunoblot for H3K79me2 demonstrating improved DOT1L inhibition by 11 in MOLM-13 cells treated for 4 days (all at 1 $\mathrm{uM})$. (g) 11 (10 uM) demonstrates specificity for H3K79me2 inhibition by immunoblot for histone methylation marks in MOLM-13 cells. (h) HoxA9 and Meis 1 mRNA in MV4;11 cells decreases with DOT1L inhibition $(10 \mathrm{uM})$ in proportion to cellular IC s0 $_{\text {after }} 7$ days of treatment (RTPCR). (i) Inhibition of MV4;11 cell growth over time with DOT1L inhibitor treatment (10 uM) demonstrates correlation with HCS potency.

dynamic range of this assay format proved capable of supporting dose-ranging studies and correctly ranked the cellular potency of all inhibitors: in comparative format, EPZ004777 was more potent than FED1, and SAH was ineffective at reducing $\mathrm{H} 3 \mathrm{~K} 79$ methylation levels in cell culture. This cellular assay allows both the comparisons between compounds to measure in-cell efficacy and the evaluation of multiple compounds simultaneously in dose-response. It is also amenable to further evaluate any screening hits obtained from the AlphaScreen and FP assays and could also be employed as a primary assay for cell-based, high-throughput screening $\left(Z^{\prime}=0.62\right)$.

To test the DOT1L discovery platform, we generated a small focused library of compounds exploring the tolerability of substitutions of the adenosine base using a highly parallel chemistry we codeveloped to explore surface-recognition features of organic ligands (hydrazine cap-scan technology). ${ }^{21,22}$ Informed by the DOT1L-FED1 and DOT1L-1 crystal structures, ${ }^{18}$ we developed a focused library of N6substituted ligands. A hydrazine functional group was introduced to the adenosine ring via a nine-step synthesis starting from known compound 3 (Supplemental Scheme 3). The hydrazine 4 then condensed with 90 divergent aldehydes/ ketones in 96-well format to generate the hydrazone library 5 (Figure 3a) without the need for further purification. The facile assembly of the library allowed rapid exploration of structureactivity relationships (SAR) at N6, and the exercise provided firm validation of the assay cascade. All analogues were evaluated in both biochemical assays in four-point dose response, followed by the H3K79me2 high content screening at a single dose. Most members of the hydrazine library showed high levels of activity in all three assays, suggesting that this site 
is highly permissive for a wide range of chemical functionalities (Figure $3 b-d$ ). Comparison of library activity in the two biochemical assays demonstrated generally good agreement between both assays (Figure S2). A group of outliers that were highly active in only the bead-based assay were triaged as likely assay-interference agents. Three of the hydrazones that showed the most consistent activity between all assays were resynthesized for retest with a 10-point, dose-response curve; these three compounds contained nitrogenous heterocyclic rings, including an indole (6), an imidazole (7), and a pyridine $(8$; Figure $3 \mathrm{e})$. While these three compounds are more potent than the parent hydrazine 4 , they had modestly weaker biochemical activity than FED1 (Figure 3f). Although the modifications explored in the focused library did not provide large gains in potency, the study validated all assays in a highthroughput format.

We then explored further features of the SAM-like core structure. In addition to the N6 substitutions, we modified the urea tail along with another location on the adenosine base. The urea motif has also been further explored with generating a small urea library (Supporting Information Scheme 5), and we discovered introduction of the thiourea group to FED1 produced a 10-fold increase in cellular activity (9) without significantly changing biochemical activity. On the adenosine base, we focused on the $\mathrm{C} 7$ position of the ring and discovered that introduction of a chlorine group on the $\mathrm{C} 7$ position of the deazaadenosine motif could generate a desirable interaction with the nearby hydrophobic pocket (10), leading to an increase in potency in all assays. By combining these two beneficial optimizations, we generated 11 with the introduction of both C7-chlorine and thiourea (Figure 4a). 11 was more potent as we expected in both the biochemical assays, and both 10 and 11 were more potent than EPZ004777 in the H3K79me2 high-content assay (Figure $4 \mathrm{~b}-\mathrm{e}$ ).

With the improved potency in the cellular assay, we then confirmed the relative potencies of these compounds by immunoblotting for H3K79me2 in MLL cells (MOLM-13). This was followed by evaluation of the selectivity of the representative 11 to inhibit only the DOT1L methyltransferase by immunoblotting for a number of histone methylation marks mediated by other KMTases and an arginine methyltransferase. The only mark affected by these compounds is H3K79me2 (Figure 4f,g, Figure S3). We then further ensured the on-target effect of these compounds by assessing gene-expression changes in MV4;11 cells. Decreased expression of both MLLtarget genes HOXA9 and MEIS1 was observed after 7 days of incubation. The potency in gene expression correlated to effects on H3K79me2 reported by high-content screening, further validating that the 4-day $\mathrm{H} 3 \mathrm{~K} 79$ me2 measurement accurately predicts on-target biological activity previously observed after 7-10 days of treatment (Figure 4h). As expected, these measurements also correlated with an antiproliferative effect in treated MV4;11 cells (Figure 4i). Therefore, utilizing our novel assay cascade and structural information, we developed inhibitors of DOT1L with enhanced cellular activity and maintained selectivity compared to previously reported compounds.

Our approach to affinity ligand design for assay development was based on a structural understanding of the binding mode between small molecule and target. Since the addition of the handle on the small molecule does not impact its DOT1L potency, the resultant probes $\mathbf{1}$ and $\mathbf{2}$ reported here can be used as chemical tools for assay development and further mechanistic studies of the DOT1L complex and its function in MLL. ${ }^{23}$ The hydrazine library demonstrated the accommodation of DOT1L to large substituents off the base, but potency was not maintained, perhaps from impurities in the original screen. However, this site appears to be permissible for future medicinal chemistry efforts toward improving pharmacokinetics or compound stability. Further exploration of the base and urea tail moiety, as accurately characterized by our assay cascade, led to the identification of more potent compounds than EPZ004777 with improved cellular activity.

\section{CONCLUSIONS}

Together, these chemical biology tools for the study of DOT1L provide a nimble platform for discovery chemistry. The labelfree biochemical assays and rapid cellular assay will be useful for discovering both allosteric and direct SAM-competitive DOT1L inhibitors, although substrate-competitive inhibitors may be silent in these biochemical assays. The high content assay, however, should be agnostic to the mode of inhibition. It also has the potential to detect inhibitors of other proteins that modulate DOT1L activity or the rate of $\mathrm{H} 3 \mathrm{~K} 79 \mathrm{me} 2$ removal. These tagged and potent inhibitors are openly available for use to probe DOT1L biology. We hope this design principle will be adapted to inhibitor discovery for other critical methyltransferases implicated in disease, including EHZ2 and MMSET.

\section{METHODS}

For protein expression and purification, crystallization, data collection and indexing, isothermal calorimetry, protein thermal melt, cell culture, gene expression, and immunoblotting, please see the Supporting Information.

DOT1L AlphaScreen Binding Assay. All reagents were diluted in $50 \mathrm{mM}$ HEPES, $150 \mathrm{mM} \mathrm{NaCl}, 0.5 \% \mathrm{BSA}(\mathrm{w} / \mathrm{v})$, $0.05 \%$ Tween $20(\mathrm{w} / \mathrm{v})$, and $\mathrm{pH} 8.0$ with $1 \mathrm{mM}$ DTT added. The final concentration of His6-DOT1L was $80 \mathrm{nM}$, and that of 1 was $40 \mathrm{nM}$. The addition of $10 \mathrm{uL}$ of $2 \times$ this solution to the plates (AlphaScreen plates, PerkinElmer \#6005359) was performed with a liquid handler. A total of $100 \mathrm{~nL}$ of compounds was added by pin transfer using a Janus Workststation (PerkinElmer, USA). After a brief centrifugation, plates were incubated at RT for $30 \mathrm{~min}$. A $2 \times$ solution of beads was made such the final concentrations of both the acceptor and donor beads were $25 \mu \mathrm{g} \mathrm{mL}^{-1}$. A total of $10 \mu \mathrm{L}$ of this solution was added to the plate, and after centrifugation and 20 min incubation, plates were read on the Envision 2104 plate reader (PerkinElmer, USA). Dose response data normalized to DMSO controls.

DOT1L Fluorescence Polarization Assay. All reagents were diluted in PBS with $1 \mathrm{mM}$ DTT freshly added. A total of 5 $\mu \mathrm{L}$ of DOT1L solution (final concentration $1 \mu \mathrm{M}$ ) was added to 384-well plates (Thermo Scientific 262260) with a Biotek EL406 liquid handler. A total of $100 \mathrm{~nL}$ of compounds from stock plates was added by pin transfer using a Janus Workststation. After a brief centrifugation, plates were incubated at RT for $30 \mathrm{~min}$. A total of $5 \mu \mathrm{L}$ of a $2 \times$ solution of 2 (final concentration $10 \mathrm{nM}$ ) was added, and the plate was briefly centrifuged. After $30 \mathrm{~min}$ incubation at RT, plates were read on the Envision 2104 plate reader. Flatfield and polarization calculations were performed by manufacturer's protocol, and anisotropy was calculated based on the formula $2 P / 3-P$ in which $P=$ polarization. Results were normalized to DMSO controls. 
High Content Imaging Assay. A431 cells were plated at 1000 cells/well in $50 \mu \mathrm{L}$ in 384-well clear bottom plates (Corning 3712) and incubated for $1 \mathrm{~h}$ at RT. Compounds were added using a Janus Workstation and incubated for 4 days. After this, cells were fixed in $3.7 \%$ formaldehyde in PBS at RT $\times 10 \mathrm{~min}$. After two rinses with blocking solution (1\% BSA in PBS), 1\% SDS in PBS was added for 2 min. After one wash, cells were incubated with blocking solution at RT $\times 30 \mathrm{~min}$. Cells were then incubated for either $1 \mathrm{~h}$ at RT or overnight at 4 ${ }^{\circ} \mathrm{C}$ in $10 \mu \mathrm{L}$ of primary antibody for $\mathrm{H} 3 \mathrm{~K} 79 \mathrm{me} 2$ (ab3594) at a 1:500 dilution in blocking solution. After rinsing with blocking solution, cells were then incubated for $1 \mathrm{~h}$ at RT in $10 \mu \mathrm{L}$ of secondary antibody (Invitrogen A-21244) and nuclear staining (Invitrogen $\mathrm{H} 3570$ ) solution at 1:1000 dilution in blocking solution. Cells were washed twice, after which $50 \mu \mathrm{L}$ of PBS was added to each well. Images were acquired on a high content screening microscope (ImageXpress Micro, Molecular Devices), and image analysis (MetaXpress3.0, Molecular Devices) was performed to obtain an average $\mathrm{H} 3 \mathrm{~K} 79 \mathrm{me} 2$ signal per cell. Dose response data (normalized to DMSO) were generated (Graphpad Prism) by normalization of maximum and minimum H3K79me2.

\section{ASSOCIATED CONTENT}

\section{(S) Supporting Information}

Figures S1-S3, supplemental methods, spreadsheet. This material is available free of charge via the Internet at http:// pubs.acs.org. The crystal structure of compound $\mathbf{1}$ bound to DOT1L has been deposited in the Protein Data Bank (PDB code: 4WVL).

\section{AUTHOR INFORMATION}

\section{Corresponding Author}

*E-mail: James_Bradner@dfci.harvard.edu.

\section{Author Contributions}

J.S.Y., A.J.F., J.Q., J.E.B., and S.A.A. designed the experiments and analyzed the data. J.S.Y., J.Q. and A.J.F. developed and optimized assays. S.D.P., R.S.P., and A.S. generated protein, and X.X. solved crystal structures. J.S.Y., M.H., and W.B.S. performed the cellular studies. J.Q., A.J.F., J.S.Y., J.J.M., E.J.C., and A.C.V. designed and synthesized all the compounds. S.A.A. provided guidance and advice. J.S.Y., A.J.F., J.Q., and J.E.B. wrote the manuscript, and all authors reviewed the manuscript.

\section{Author Contributions}

These authors contributed equally to the work.

\section{Notes}

The authors declare the following competing financial interest(s): SAA is a consultant for Epizyme, Inc.

\section{ACKNOWLEDGMENTS}

We thank R. Paranal for his assistance in developing the high content assay; we thank C. Ott for helpful discussions and D. Buckley for thoughtful review of the manuscript. This work was funded by the U.S. National Institutes of Health grant R01 CA176745, the William Lawrence \& Blanche Hughes Foundation, the Leukemia \& Lymphoma Society SCOR grant, and the American Society of Hematology-Scholar Award.

\section{REFERENCES}

(1) Krivtsov, A., and Armstrong, S. (2007) MLL translocations, histone modifications and leukaemia stem-cell development. Nat. Rev. Cancer 7, 823-833.
(2) Goodell, M. (2013) Epigenetics in hematology: introducing a collection of reviews. Blood 121, 3059-3060.

(3) Neff, T., and Armstrong, S. A. (2013) Recent progress toward epigenetic therapies: the example of mixed lineage leukemia. Blood $121,4847-4853$.

(4) Steger, D., Lefterova, M., Ying, L., Stonestrom, A., Schupp, M., Zhuo, D., Vakoc, A., Kim, J.-E., Chen, J., Lazar, M., Blobel, G., and Vakoc, C. (2008) DOT1L/KMT4 recruitment and H3K79 methylation are ubiquitously coupled with gene transcription in mammalian cells. Mol. Cell Biol. 28, 2825-2839.

(5) Guenther, M., Lawton, L., Rozovskaia, T., Frampton, G., Levine, S., Volkert, T., Croce, C., Nakamura, T., Canaani, E., and Young, R. (2008) Aberrant chromatin at genes encoding stem cell regulators in human mixed-lineage leukemia. Genes Dev. 22, 3403-3408.

(6) Bernt, K., Zhu, N., Sinha, A., Vempati, S., Faber, J., Krivtsov, A., Feng, Z., Punt, N., Daigle, A., Bullinger, L., Pollock, R., Richon, V., Kung, A., and Armstrong, S. (2011) MLL-rearranged leukemia is dependent on aberrant H3K79 methylation by DOT1L. Cancer Cell. 20, 66-78.

(7) Bitoun, E., Oliver, P., and Davies, K. (2007) The mixed-lineage leukemia fusion partner AF4 stimulates RNA polymerase II transcriptional elongation and mediates coordinated chromatin remodeling. Hum. Mol. Genet. 16, 92-106.

(8) Krivtsov, A., Feng, Z., Lemieux, M., Faber, J., Vempati, S., Sinha, A., Xia, X., Jesneck, J., Bracken, A., Silverman, L., Kutok, J., Kung, A., and Armstrong, S. (2008) H3K79 methylation profiles define murine and human MLL-AF4 leukemias. Cancer Cell 14, 355-368.

(9) Okada, Y., Feng, Q., Lin, Y., Jiang, Q., Li, Y., Coffield, V., Su, L., $\mathrm{Xu}, \mathrm{G}$., and Zhang, Y. (2005) hDOT1L links histone methylation to leukemogenesis. Cell 121, 167-178.

(10) Deshpande, A., Chen, L., Fazio, M., Sinha, A., Bernt, K., Banka, D., Dias, S., Chang, J., Olhava, E., Daigle, S., Richon, V., Pollock, R., and Armstrong, S. (2013) Leukemic transformation by the MLL-AF6 fusion oncogene requires the H3K79 methyltransferase Dotll. Blood $121,2533-2541$.

(11) Jo, S. Y., Granowicz, E. M., Maillard, I., Thomas, D., and Hess, J. L. (2011) Requirement for Dot1l in murine postnatal hematopoiesis and leukemogenesis by MLL translocation. Blood 117, 4759-4768.

(12) Nguyen, A. T., Taranova, O., He, J., and Zhang, Y. (2011) DOT1L, the H3K79 methyltransferase, is required for MLL-AF9mediated leukemogenesis. Blood 117, 6912-6922.

(13) Daigle, S., Olhava, E., Therkelsen, C., Majer, C., Sneeringer, C., Song, J., Johnston, L., Scott, M., Smith, J., Xiao, Y., Jin, L., Kuntz, K., Chesworth, R., Moyer, M., Bernt, K., Tseng, J.-C., Kung, A., Armstrong, S., Copeland, R., Richon, V., and Pollock, R. (2011) Selective killing of mixed lineage leukemia cells by a potent smallmolecule DOT1L inhibitor. Cancer Cell. 20, 53-65.

(14) Yu, W., Chory, E., Wernimont, A., Tempel, W., Scopton, A., Federation, A., Marineau, J., Qi, J., Barsyte-Lovejoy, D., Yi, J., Marcellus, R., Iacob, R., Engen, J., Griffin, C., Aman, A., Wienholds, E., Li, F., Pineda, J., Estiu, G., Shatseva, T., Hajian, T., Al-Awar, R., Dick, J., Vedadi, M., Brown, P., Arrowsmith, C., Bradner, J., and Schapira, M. (2012) Catalytic site remodelling of the DOT1L methyltransferase by selective inhibitors. Nat. Commun. 3, 1-11.

(15) Daigle, S., Olhava, E., Therkelsen, C., Basavapathruni, A., Jin, L., Boriack-Sjodin, P., Allain, C., Klaus, C., Raimondi, A., Scott, M., Waters, N., Chesworth, R., Moyer, M., Copeland, R., Richon, V., and Pollock, R. (2013) Potent inhibition of DOT1L as treatment of MLLfusion leukemia. Blood 122, 1017-1025.

(16) Deng, L., Zhang, L., Yao, Y., Wang, C., Redell, M., Dong, S., and Song, Y. (2013) Synthesis, Activity and Metabolic Stability of NonRibose Containing Inhibitors of Histone Methyltransferase DOT1L. MedChemComm. 4, 822-826.

(17) Yao, Y., Chen, P., Diao, J., Cheng, G., Deng, L., Anglin, J., Prasad, B. V., and Song, Y. (2011) Selective inhibitors of histone methyltransferase DOT1L: design, synthesis, and crystallographic studies. J. Am. Chem. Soc. 133, 16746-16749.

(18) Anglin, J., Deng, L., Yao, Y., Cai, G., Liu, Z., Jiang, H., Cheng, G., Chen, P., Dong, S., and Song, Y. (2012) Synthesis and structure- 
activity relationship investigation of adenosine-containing inhibitors of histone methyltransferase DOT1L. J. Med. Chem. 55, 8066-8074.

(19) Yu, W., Smil, D., Li, F., Tempel, W., Fedorov, O., Nguyen, K., Bolshan, Y., Al-Awar, R., Knapp, S., Arrowsmith, C., Vedadi, M., Brown, P., and Schapira, M. (2013) Bromo-deaza-SAH: a potent and selective DOT1L inhibitor. Bioorg. Med. Chem. 21, 1787-1794.

(20) McGinty, R. K., Köhn, M., Chatterjee, C., Chiang, K. P., Pratt, M. R. and Muir, T. W. (2009) Structure-activity analysis of semisynthetic nucleosomes: mechanistic insights into the stimulation of Dot1L by ubiquitylated histone H2B. ACS Chem. Biol. 4, 958-968.

(21) Vegas, A., Bradner, J., Tang, W., McPherson, O., Greenberg, E., Koehler, A., and Schreiber, S. (2007) Fluorous-based small-molecule microarrays for the discovery of histone deacetylase inhibitors. Angew. Chem., Int. Ed. Engl. 46, 7960-7964.

(22) Bradner, J., West, N., Grachan, M., Greenberg, E., Haggarty, S., Warnow, T., and Mazitschek, R. (2010) Chemical phylogenetics of histone deacetylases. Nat. Chem. Biol. 6, 238-243.

(23) Anders, L., Guenther, M., Qi, J., Fan, Z., Marineau, J., Rahl, P., Lovén, J., Sigova, A., Smith, W., Lee, T., Bradner, J., and Young, R. (2014) Genome-wide localization of small molecules. Nat. Biotechnol. $32,92-96$. 